\title{
Modification of the strange meson properties in nuclear matter
}

\author{
Yury Kiselev ${ }^{1, \star}$, Michael Hartmann ${ }^{2}$, Eduard Paryev ${ }^{1,3}$, and Andrey Polyanskiy ${ }^{1}$ \\ ${ }^{1}$ Institute for Theoretical and Experimental Physics, Moscow, Russia \\ ${ }^{2}$ Institute for Nuclear Physics, FZ-Juelich, Juelich, Germany \\ ${ }^{3}$ Institute for Nuclear Research, Russian Academy of Science, Moscow, Russia
}

\begin{abstract}
To make progress in understanding the properties of the strange mesons with open $\left(K^{-}\right)$and hidden $(\phi)$ strangeness in the nuclear medium, the measurements of the production of $K^{+} K^{-}$pairs with invariant masses corresponding to both the $\phi$ and non- $\phi$ regions in proton collisions with $\mathrm{C}, \mathrm{Cu}, \mathrm{Ag}$, and $\mathrm{Au}$ targets at an incident beam energy of $2.83 \mathrm{GeV}$ were recently performed by the ANKE Collaboration at COSY. The data from the $\phi$ region of invariant masses provide information on the momentum dependence of the $\phi$ nuclear transparency ratio and the in-medium $\phi$ meson width over the momentum range of $0.6-1.6 \mathrm{GeV} / \mathrm{c}$. Analysis of the data from the non- $\phi$ region of $K^{+} K^{-}$invariant masses, where differential cross sections for pair production on the four targets were obtained as functions of the laboratory $K^{-}$momentum, allows to draw the conclusion of the strength of the $K^{-}$-nuclear potential.
\end{abstract}

\section{Introduction}

The study of hadron properties in a strongly interacting environment has been a very active research field over the last years, especially in connection with questions of the partial restoration of chiral symmetry in hot/dense nuclear matter and of the existence of a $K^{-}$condensate in neutron stars. Modification of hadron properties, mass and width, has been predicted by the QCD sum rules and the models with chiral Lagrangians. As the strength of the effects is expected to be proportional to the baryon density and/or the temperature, the main efforts over the last decades were focused at the quests for these effects in the relativistic heavy-ion collisions. However, the difficulties associated with the problem of the description of system with a drastic change in time its density and temperature did not allow to make the final conclusion about the existence of the modification effects. An alternative way to get the information on the hadron medium modification effects is the study of the reaction with elementary probes (photons, pions, and protons) on the nuclei. The advantage of this approach is connected to the fact that the process of hadron production proceeds in cold static nuclear matter of a well-defined density while the values of the effects are somewhat less but still comparable to those of heavy-ion collisions. At normal nuclear density, $\rho_{0}=0.16 \mathrm{fm}^{-3}$, the in-medium modification of mesons can be expressed in terms of complex nuclear optical potential U. In this case the BreitWigner formula acquires two new terms: $S(M)=\left[\Gamma_{0} / 2-\operatorname{Im} U\right] /\left[\left(M-\left(M_{0}+\operatorname{Re} U\right)\right)^{2}+\left(\Gamma_{0} / 2-\operatorname{Im} U\right)^{2}\right]$. The real part of the potential $\operatorname{Re} U$ determines a shift of the pole of the meson spectral function while

\footnotetext{
^e-mail: yurikis@itep.ru
} 
the imaginary part $\operatorname{Im} U$ determines its broadening in nuclear matter. It is expected that both parts of the potential $U$ depend on the meson momentum relative to the nuclear matter. Here we report on the results of the investigation of the in-medium properties of the mesons with hidden $(\phi)$ and open $\left(K^{-}\right)$strangeness produced by $2.83 \mathrm{GeV}$ proton beam on nucleus targets. According to the theoretical predictions a decrease of the $\phi$ meson mass at normal nuclear density is small $(<1 \%)$ and the main medium effect on the $\phi$ is substantial increase of its width compared to its vacuum magnitude of 4.43 $\mathrm{MeV}$. This magnitude corresponds to the decay length $L=46 \mathrm{fm}$ what significantly exceeds the size of nuclei. In spite of almost all produced $\phi$ 's decay into $K^{+} K^{-}$pairs outside the nucleus, the study of the $\phi$ absorption inside the nuclei provides a possibility to derive the imaginary part of the nuclear optical potential and, hence, the $\phi$ width $\Gamma=-2 \operatorname{Im} U$ in nuclear matter [1]. The study of the nonresonant (direct) $K^{-}$mesons production gives the opportunity to deduce the magnitude of the real part of the $K^{-}$-nuclear potential. In this case, in contrast with the $\phi$, the $K^{-}$creation occurs inside nuclei and the surrounding nuclear medium affects the properties of produced mesons.

\section{Experiment}

The experiment was performed at the COoler SYnchrotron (COSY) of the Forschungszentrum Juelich (Germany) using the ANKE magnetic spectrometer that is located at an internal target station of the storage ring. ANKE contains three dipole magnets; D1 and D3 divert the circulating beam onto the target and back into the COSY ring, respectively, while D2 is the analyzing magnet [2]. A series of thin and narrow $\mathrm{C}, \mathrm{Cu}, \mathrm{Ag}$, and $\mathrm{Au}$ targets was inserted in a circulating beam of $2.83 \mathrm{GeV}$ protons in front of the main spectrometer magnet D2. The ANKE spectrometer has detection systems placed to the right and left of the beam to register positively and negatively charged ejectiles which are the $\mathrm{K}^{+}$and $\mathrm{K}^{-}$in the case of the present study. Although only used here for efficiency studies, forwardgoing charged particles could also be measured in coincidence. The spectrometer is equipped by the proportional chambers for tracking, the scintillation counters for the time-of-flight and energy loss measurements, and Cherenkov counter for the pion suppression. The accessible ranges of the $K^{+}$ and $K^{-}$meson momenta were $0.2<\mathrm{p}<0.6 \mathrm{GeV} / \mathrm{c}$ and $0.2<\mathrm{p}<1.0 \mathrm{GeV} / \mathrm{c}$, respectively. The polar production angle was restricted to $12^{0}$ both for positively and negatively charged kaons. The initial proton kinetic energy of $2.83 \mathrm{GeV}$ corresponds to the excess energy of $108 \mathrm{MeV}$ above the threshold for the kaon pair creation in proton-nucleon collisions. The integrated luminosity for each target is calculated using the measured flux of $\pi^{+}$mesons with momenta of $500 \mathrm{MeV} / \mathrm{c}$ produced at small laboratory angles. Knowing the cross section of this reaction [3] allowed calculation of the differential cross sections for the kaon pair production. The positively charged kaon was first selected using a dedicated detection system that can identify a $K^{+}$against a pion and/or proton background that is $10^{5}$ times more intense [4], [5]. The coincident $K^{-}$was subsequently identified from the timeof-flight difference between the stop counters in the negative and positive detector systems [6].

\section{Analysis of data}

The resulting invariant mass spectrum of the selected $K^{+} K^{-}$pair for $\mathrm{C}$ is given in figure 1 . One can see that there is strong $\phi$ signal that sits on a broad distribution of non-resonant kaon pair production together with a relatively small number of misidentified events. To separate the non-resonant kaon pair production events from those arising from the $\phi$ resonance, a cut on the invariant mass of $\mathrm{K}^{+} \mathrm{K}^{-}$ pairs, IM $<1.005 \mathrm{GeV} / \mathrm{c}^{2}$, was applied in the subsequent analysis. To determine the in-medium $\phi$ meson width a $3 \sigma$ cut around the $\phi$ peak was made to select $K^{+} K^{-}$events steaming from the $\phi$ decay. For each of the four targets this part of mass spectrum was fitted by the incoherent sum of a BreitWigner function with the natural $\phi$ width, convoluted with Gaussian resolution function with $\sigma=1$ 


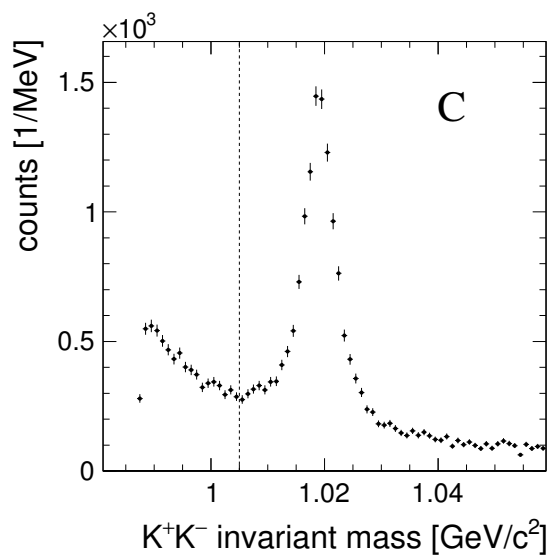

Figure 1. Invariant mass (IM) distribution for $K^{+} K^{-}$pair produced in $\mathrm{pC}$ collisions at $2.83 \mathrm{GeV}$ beam energy. The vertical line indicates the cut $\mathrm{IM}=1.005 \mathrm{GeV} / \mathrm{c}^{2}$ used for the separation of the non-resonant (left) and resonant (right) production processes.

$\mathrm{MeV}$, and polynomial background function. Since the acceptance and efficiency corrections in ANKE are essentially target-independent, the ratio of the numbers of reconstructed $\phi$ mesons corresponds to the ratio of the cross sections for $\phi$ meson production in $\mathrm{pA}$ and $\mathrm{pC}$ collisions in the ANKE window. Values of the nuclear transparency ratio normalized to carbon, $R=(12 / A)\left(\sigma_{\phi}^{A} / \sigma_{\phi}^{C}\right)$, averaged over the $\phi$ momentum range $0.6-1.6 \mathrm{GeV} / \mathrm{c}$, are presented in our work [7]. The number of reconstructed $\phi$ mesons for each target was between 7000 and 10000.

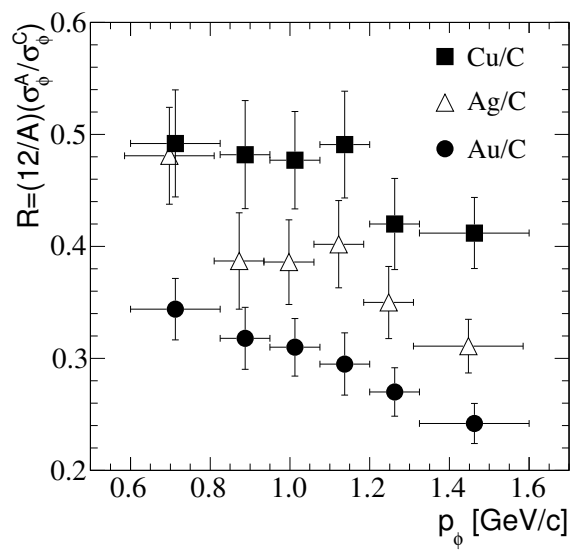

Figure 2. Momentum dependence of the transparency ratio $\mathrm{R}$, normalized to carbon, for $\mathrm{Cu}, \mathrm{Ag}$, and $\mathrm{Au}$ targets.

These data allowed the momentum dependence of $\phi$ nuclear transparency, the in-medium $\phi$ width to be determined for four targets. The transparency ratio for $\mathrm{Au}, \mathrm{Ag}$, and $\mathrm{Cu}$ targets as a function of the $\phi$ laboratory momentum $p_{\phi}$ is shown in figure 2 . The values of the transparency ratio can be measured more precisely than the cross sections themselves as the uncertainties in the absolute normalization and the acceptance calculations as well as the detector-dependent errors mostly cancel out in the ratio. The momentum dependence of the $\phi$ meson width has been extracted from the comparison of the measured transparency ratios with those calculated in frame of different approaches. We consider three models. The first model is based on the chiral invariant approach elaborated by the Valencia group [1], second one relayed on the BUU transport calculations of the Rossendorf group [8] and the third model is based on the spectral function approach developed by E. Paryev [9]. By comparing the calculated and measured values of the transparency ratio for three target combinations, it is possible 


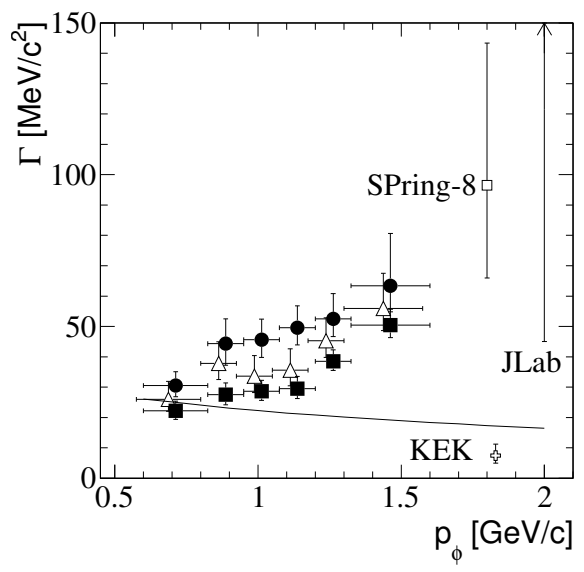

Figure 3. In-medium width of the $\phi$ meson in the nuclear rest frame at saturation density $\rho_{0}$ as a function of the $\phi$ momentum. The points have been evaluated by comparing the data of figure 2 with the results of three model calculations (model [1]-solid squares, model [9]-solid circles and model [8]-open triangles). Also shown are the results from the other experiments [11], [12], [13]. The solid line represents the $\Gamma_{\phi}$ calculated on the basis of the predicted $\phi$ self-energy in nuclear matter [14].

Table 1. The $\phi$ meson width $\Gamma\left[\mathrm{MeV} / \mathrm{c}^{2}\right]$ at the laboratory momentum $p_{\phi}[\mathrm{GeV} / \mathrm{c}]$. The results of the calculation assuming no medium effects on the $\phi$ width and the mean values of the $\phi$ meson width shown in figure 3 are presented in the second and third lines, respectively.

\begin{tabular}{lllllll}
\hline$p_{\phi}$ & 0.71 & 0.89 & 1.01 & 1.14 & 1.26 & 1.42 \\
\hline$\Gamma_{\text {vac }}$ & 16.6 & 18.2 & 19.0 & 19.8 & 20.3 & 21.0 \\
$\Gamma$ & 26 & 34 & 37 & 40 & 45 & 54 \\
\hline
\end{tabular}

to determine the weighted average of the $\phi$ width $\Gamma$ for each momentum bin in the nuclear rest frame for density $\rho_{0}$. The result of applying this procedure is presented in figure 3 . As it can be seen from figure 3 the application of three models yields broadly consistent results. Observed significant increasing width with momentum does not depend on the applied model.

The total in-medium $\phi$ width $\Gamma$ comprises decay and collision components. The value of both components can be modified due to the interaction of the $\phi$ with nuclear environment. In order to draw conclusion whether the observed $\phi$ width exhibits non-trivial medium effects, one has to compare this with the case of no influence of the surrounding matter on the $\phi$ properties. To do so we calculated $\Gamma_{v a c}=\Gamma_{d e c}+\Gamma_{c o l}$ for the center momentum of each momentum bin assuming no medium effects on the $\phi$ width. The calculations were performed using the vacuum value of the $\Gamma_{d e c}=4.43 \mathrm{MeV} / \mathrm{c}^{2}$ from PDG and the value of the $\Gamma_{c o l}$ deduced in the low-density approximation $\Gamma_{c o l}=\beta \rho_{0} \sigma_{v a c}(\phi N)$. Here $\beta=p / E$ is the $\phi$ meson velocity relative to the nuclear matter having a density of $\rho_{0}$, while $\sigma_{v a c}(\phi N)$ is the mean vacuum $\phi$-nucleon cross section of $9 \mathrm{mb}$. The latter value is known from the analysis of the experiments on the $\phi$ photoproduction from proton and it is in line with the prediction of the additive quark model. The results of the calculations are presented in table 1 together with mean values of the width $\Gamma$ shown in figure 3. Comparison of the second and third lines of the table provides the clear evidence for the modification of the $\phi$ width in strongly interacting environment already at normal nuclear density.

The question about the modification of the $K^{-}$meson properties in nuclear matter are still the subject of very intense debate. This is due to the complicated dynamics of antikaons inside nuclei, which leads to modifications of their in-medium properties. The calculations, based on chiral Lagrangians or on meson-exchange potentials, predict relatively shallow low-energy $K^{-}$-nucleus potentials with central depths of the order of -50 to $-80 \mathrm{MeV}$. On the other hand, fits to the $K^{-}$atomic data, in terms of phenomenological density dependent optical potentials or relativistic mean-field calculations, lead to much stronger potentials with depths of about $-200 \mathrm{MeV}$ at density $\rho_{0}$. Current experimental esti- 
mates of the potential are spread over the wide range from $-30 \mathrm{MeV}$ to $-200 \mathrm{MeV}$. To make progress in understanding the strength of the $K^{-}$interaction in the nuclear medium we studied the properties of the $K^{-}$produced together with $K^{+}$mesons on nuclear targets in the non-resonant process. Such events form low mass part of the invariant mass spectrum IM $<1.005 \mathrm{GeV} / \mathrm{c}^{2}$ (see figure 1). In contrast to the inclusive measurements, this data set does not contain kaon pairs steaming from the $\phi$ decay outside the nuclei. For further interpretation of the data on non-resonant $K^{-}$meson production the corre-

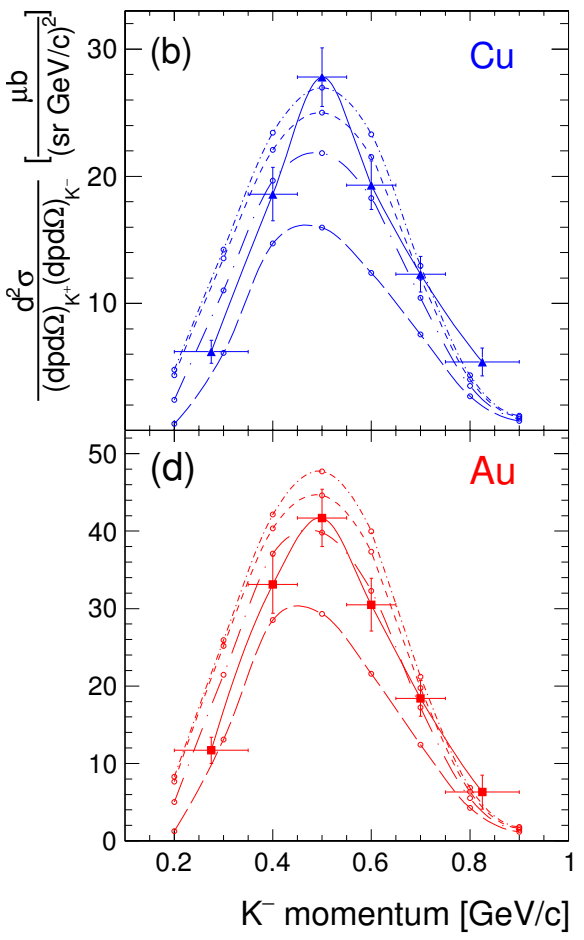

Figure 4. Double-differential cross sections for the production of non-resonant $K^{+} K^{-}$pairs on $\mathrm{Cu}$, (b), and $\mathrm{Au}$ (d) targets as a function of the $K^{-}$laboratory momentum. The experimental data are averaged over $K^{+}$momenta in the range $200<p_{K^{+}}<600 \mathrm{MeV} / \mathrm{c}$. The curves represent, from bottom to top, model calculations [10] for $K^{-}$potential depth $U=0$ $\mathrm{MeV}$ (long dashed), $-60 \mathrm{MeV}$ (dot long dashed), $-126 \mathrm{MeV}$ (short dashed), and -180 MeV (dot short dashed), respectively. The solid lines are simple spline functions through the experimental points.

sponding differential cross section were calculated in the frame of the collision model, based on the nuclear spectral function [10], assuming four options for the $K^{-}$nuclear potential depth at normal nuclear matter density, viz. $U=0 \mathrm{MeV}, U=-60 \mathrm{MeV} U=-126 \mathrm{MeV}$, and $U=-180 \mathrm{MeV}$. The calculations take into account the ANKE acceptance. An example of the performed calculations shown in figure 4 demonstrates rather strong sensitivity of the cross sections to the depth of the potential and, therefore, can be used for its determination.

In the following analysis of the data, aiming at the determination of the real part of the antikaon nuclear potential at saturation density, we make use of the cross sections integrated over the measured $K^{-}$momentum interval $0.2-1.0 \mathrm{GeV} / \mathrm{c}$. Due to the increased number of counts, this approach has the advantage of decreasing significantly the statistical and partially systematic uncertainties. Evidently, the antikaon potential depth extracted in this way will correspond to an average $K^{-}$momentum of about $0.5 \mathrm{GeV} / \mathrm{c}$, in the vicinity of which the main strength of the measured distributions is concentrated. The calculations of the corresponding cross sections were performed within the model for different potential strengths. The values of $\sigma_{\text {exp }} / \sigma_{\text {cal }}(U)$ are shown in figure 5 for $U=0 \mathrm{MeV}, U=$ $-60 \mathrm{MeV}, U=-126 \mathrm{MeV}$, and $U=-180 \mathrm{MeV}$. Also shown is a third-order polynomial fit to the complete data set of ratios. It is seen from the figure that the condition that $\sigma_{\text {exp }} / \sigma_{\text {cal }}(U)=1$ is achieved if $U=-63_{-12}^{+15} \mathrm{MeV}$. This means that the $K^{-}$effective mass in the nuclear matter of normal density is 


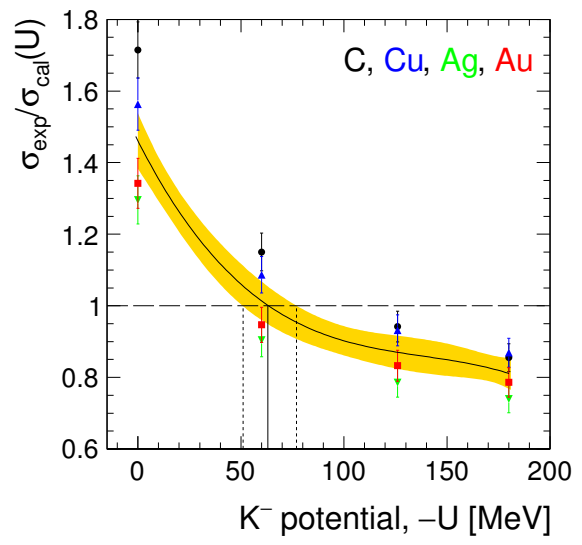

Figure 5. Ratio of the measured integrated cross section for the non-resonant $K^{+} K^{-}$production by protons on a given nucleus A to the corresponding cross sections, calculated within the adopted model and supposing four values for the $K^{-}$potential depth at nuclear matter density: $U=0 \mathrm{MeV},-60$ $\mathrm{MeV},-126 \mathrm{MeV},-180 \mathrm{MeV}$. The curve represents a third-order polynomial fit of all ratios presented in the figure, with the shaded band indicating $1 \sigma$ confidence interval.

less than its free mass by about of $60 \mathrm{MeV}$. Acting on the cautious side and using the maximum value of the final systematic uncertainty we came to the expanded error band of $U=-63_{-31}^{+50} \mathrm{MeV}$. Within the uncertainties quoted, the value obtained for the potential depth is consistent with the moderate $K^{-}$nucleus potential that is predicted by calculations based on chiral Lagrangians or on meson-exchange potentials. However, it is hard to reconcile our value with the deep potential of order $-200 \mathrm{MeV}$.

\section{Conclusion}

The data analysis of the performed experiment on the $K^{+} K^{-}$pair production off nuclei evidences for the properties of mesons with open $\left(K^{-}\right)$and hidden $(\phi)$ strangeness in the nuclear matter significantly differ from those in the free space.

\section{References}

[1] V. K. Magas et al., Phys. Rev. C 71, 065202 (2005)

[2] S. Barsov et al., Nucl. Instrum. Methods Phys. Res., Sect. A 462, 364 (2010)

[3] M. Hartmann et al., Phys. Rev. C 85, 035206 (2012)

[4] M. Hartmann et al., Int. J. Mod. Phys. A 22, 317 (2007)

[5] M. Buesher et al., Nucl. Instrum. Methods Phys. Res., Sect. A 481, 378 (2002)

[6] Yu. T. Kiselev et al., Phys. Rev. C 92, 065201 (2015)

[7] A. Polyanskiy et al., Phys. Lett. B 695, 74 (2011)

[8] H. Schade, Ph.D. thesis, University of Dresden, 2010 (unpublished)

[9] E. Ya. Paryev, J. Phys. G 36, 015103 (2009)

[10] E. Ya. Paryev, M. Hartmann, and Yu. T. Kiselev, J. Phys. G 42, 0175107 (2015)

[11] R. Muto et al., Phys. Rev. Lett. 98, 042501 (2007)

[12] T. Ishikawa et al., Phys. Lett. B 608, 215 (2005)

[13] M. H. Wood et al., Phys. Rev. Lett. 105, 112301 (2010)

[14] D. Cabrera et al., Nucl. Phys. A 733, 130 (2004) 